\title{
Syphilis sero-positivity in recently admitted and long-term psychiatric inpatients: Screening, prevalence and diagnostic profile
}

\author{
M P Henning, C Krüger, L Fletcher \\ Department of Psychiatry, University of Pretoria \\ M P Henning, MB ChB, MMed (Micro), MMed (Psych), FCPsych (SA) \\ C Krüger, MB BCh, MMed (Psych), MD, FCPsych (SA) \\ Department of Statistics, University of Pretoria \\ L Fletcher, BCom, BSc (Hons), MSc, PhD
}

Correspondingauthor: C Krüger (christa.kruger@up.ac.za)

Background. Syphilis research has neglected the prevalence of the disease among psychiatric patients, and traditional syphilis screening has been reported as inadequate.

Objectives. (i) To assess the syphilis prevalence among psychiatric patients; (ii) to compare psychiatric diagnoses of syphilis-infected and -uninfected patients; (iii) to assess self-reported high-risk sexual behaviour; (iv) to establish syphilis/HIV co-morbidity; and ( $v$ ) to investigate the performance of the rapid plasma reagin (RPR) test in syphilis screening, compared with the Treponema pallidum haemagglutination (TPHA) test.

Methods. Psychiatric inpatients at Weskoppies Hospital, Pretoria, who consented to participate in the study $(N=195)$ were categorised according to gender and length of admission (long-term or recent). Non-treponemal RPR, confirmatory TPHA, HIV-rapid and HIV enzyme-linked immunosorbent assay (ELISA) tests were performed. A reactive TPHA test was used to diagnose syphilis.

Results. The estimated prevalence of syphilis was $11.7 \%$. There was no significant association between TPHA sero-positivity and primary psychiatric diagnosis or self-reported high-risk sexual behaviour. Significant co-morbidity existed between syphilis and HIV ( $p=0.012$ ). Compared with the TPHA test, the RPR test performed poorly, identifying only $2 / 23$ patients who had a sero-positive TPHA test ( $8.7 \%$ sensitivity and $100 \%$ specificity).

Conclusions. The prevalence of syphilis was higher than anticipated, supporting the need for routine testing. The significant co-morbidity and alarming prevalence of HIV and syphilis warrant testing for both conditions in all psychiatric admissions. Current syphilis screening with a single RPR test is inadequate; both RPR and TPHA tests should be performed.

S Afr J Pscyh 2012;18(4):171-175. DOI:10.7196/SAJP.358

Syphilis has an historical association with mental illness, and neurosyphilis remains relevant in the psychiatric population, inter alia, as a cause or aggravating factor of various psychiatric disorders (dementia, mood disorders, personality changes and psychosis)..$^{1-3}$ Clinical differentiation between a primary psychiatric disorder and the psychiatric symptoms of syphilis may be very difficult. ${ }^{4}$

Neuropathologically speaking, syphilis is a sexually transmitted acute or chronic infection caused by the spirochete Treponema pallidum. Disease manifestations are diverse, occurring in stages: primary, secondary, latent and tertiary (late). Neurosyphilis is regarded as a manifestation of tertiary syphilis, but the spirochete may invade the central nervous system early in the course of infection. ${ }^{5,6}$ Neurosyphilis may be asymptomatic or symptomatic, manifesting in the latter as syphilitic meningitis, meningovascular syphilis, or parenchymatous neurosyphilis.?

Because different pathological findings overlap extensively, it has been suggested that the pathological classification of neurosyphilis should be replaced with a clinical classification, e.g. neurosyphilis with neuropsychiatric disorders such as psychosis, delirium or dementia; neurosyphilis with cerebrovascular accidents; and neurosyphilis with ocular- or spinal cord involvement, epilepsy and brainstem/cranial nerve involvement. ${ }^{8}$

Epidemiologically, syphilis has been linked to HIV infection, with an associated rising incidence. ${ }^{5,7,9,10}$ In 2008 the World Health Organization (WHO) estimated there to be 10.6 million new cases of syphilis worldwide, with 36.4 million adults infected. In Africa the incidence of syphilis was estimated to be 9.4 and 8.5 per 1000 for males and females, respectively, whereas the prevalence was estimated to be 3.9 and 3.5 per 1000 for males and females, respectively. ${ }^{11}$

Only scant data are available on the prevalence of syphilis among patients with mental illness, with reported figures of $3-76 \%{ }^{9,12-14}$ In a study by Carey et al., ${ }^{13}$ bivariate associations between infection status and patient characteristics including age, gender and psychiatric 
diagnosis, did not reveal any consistent risk profile. ${ }^{13}$ Rather, infection status was associated with behavioural characteristics (multiple partners, exchanging sex for money and engagement in anal sex). ${ }^{13}$

In South Africa, research on the prevalence of syphilis in psychiatric patients is especially scarce. Most sero-prevalence data are collected from sexually transmitted disease (STD) and antenatal clinics, with tremendous variation between groups: from $6.5 \%$ in pregnant rural women to $42 \%$ in female sex-workers in KwaZulu-Natal. ${ }^{15,16}$

HIV/syphilis co-morbidity is well established. Individuals with syphilis are at increased risk of acquiring HIV, and syphilis is an important co-factor in facilitating HIV transmission. ${ }^{1718} \mathrm{Co}$-infection is common because of the shared risk factors related to sexual behaviour and the pathological changes caused by both diseases. Syphilis-induced genital tract inflammation and/or ulcerations may disrupt innate barriers to HIV. By altering normal immune responses, HIV may in turn affect the presentation, diagnosis and natural course of syphilis. ${ }^{19}$ There is also evidence that HIV may lead to more rapid progression to neurosyphilis. ${ }^{18,20}$ It is therefore important to exclude syphilis and HIV in a patient presenting with a neuropsychiatric disorder; all patients diagnosed with syphilis should be tested for HIV and vice versa. ${ }^{5,6,21}$ Unfortunately, screening tests for syphilis, e.g. the rapid plasma reagin (RPR) test, are unreliable in detecting the disease in psychiatric patients. ${ }^{9,22}$

\section{Serological diagnosis}

Although the serological diagnosis of syphilis is less than ideal, it remains the mainstay of diagnosis because $T$. pallidum cannot be cultured routinely. Traditional screening algorithms include a nontreponemal serological test, such as the RPR or Venereal Disease Research Laboratory (VDRL) titre, after which a reactive specimen is confirmed with a specific treponemal test such as the T. pallidum haemagglutination (TPHA) test. ${ }^{23}$

Non-treponemal tests measure $\operatorname{IgM}$ and $\operatorname{IgG}$ antibodies to nonspecific antigens, such as lipoidal material released from damaged host cells and lipoprotein-like material released from the treponemes. The anti-lipoidal antibodies can be produced as a consequence of syphilis, but also during pregnancy, auto-immune diseases and other chronic or acute conditions where tissue damage occurs. ${ }^{24}$ Owing to this non-specificity, reactivity of a specific treponemal test is required to confirm the diagnosis of syphilis.

Non-treponemal tests are also limited in that they lack the sensitivity to detect very early and late syphilis. Non-treponemal antibody titres peak during secondary syphilis and gradually decline; therefore, up to $20-25 \%$ of untreated late latent syphilis may be undetected by a non-treponemal antibody test. ${ }^{18}$ Nonetheless, such tests are widely available, inexpensive, quick and easy to perform, and have been used for diagnostic purposes in STD and antenatal clinics where same-day diagnosis is desired. Quantitative non-treponemal tests are also used to monitor responses to treatment (decline in titre) or to indicate new infections (increase in titre). ${ }^{7,24}$

The TPHA test detects antibodies against a specific treponemal antigen. It has a high specificity for syphilis infection, especially during the later stages of the disease, but has historically been considered too labourintensive and expensive as a first-line test. It therefore follows that some cases of syphilis infection will be missed when diagnosis is based solely on a non-treponemal test. Reeves et al. ${ }^{9}$ found a $25 \%$ prevalence of syphilis among 200 patients with chronic mental illness, with a $21 \%$ of non-detection by the RPR test. Their recommendation was to use specific treponemal tests to screen for and diagnose syphilis in mentally ill patients, instead of sole reliance on non-specific treponemal tests. ${ }^{9}$

The TPHA test measures lifetime exposure to T. pallidum infection but has only moderate sensitivity to detect early primary disease. After the test becomes reactive during the later primary and secondary stages of syphilis, it remains reactive, despite adequate antimicrobial treatment, and so can neither differentiate between current and past infection nor be used to monitor treatment success. In general, both non-treponemal and treponemal tests can be interpreted in the usual manner for patients co-infected with syphilis and HIV, although false-positive and -negative results have been described. ${ }^{23-25}$ Ideally, the specific treponemal test should be used in conjunction with the non-treponemal test, since both are needed to assess the patient's current status of infection (active or latent) and to monitor treatment success in active syphilis.

Although patients with non-reactive non-treponemal and reactive treponemal serology are unlikely to be infectious, they are at risk of already having or of developing neurosyphilis, as well as the other serious sequelae of tertiary syphilis. Whether or not routine lumbar punctures should be done to exclude neurosyphilis is a contentious issue. Studies on the serological status of patients with neurosyphilis have shown conflicting results. Some studies have reported that the majority of such patients have a non-reactive non-treponemal serum test; however, more recent studies have shown that it is unlikely for neurosyphilis patients to have a non-reactive non-treponemal serum test. ${ }^{8,26-28}$ Marra et al. ${ }^{20}$ found that neurosyphilis was significantly more common when the serum RPR titre was $>1: 32$, although up to onethird of patients with neurosyphilis had lower titres. ${ }^{20}$

Screening for active syphilis with a non-treponemal test is considered to be a baseline investigation in newly admitted psychiatric patients. A specific treponemal test is often only performed to confirm a reactive screening test. ${ }^{6,23}$ However, the RPR result alone does not give a comprehensive picture of syphilis exposure and infection. If the aim is to diagnose previously undetected tertiary infections now presenting as neurosyphilis with psychiatric symptoms, or latent infections that may progress to neurosyphilis, then a specific treponemal test should be performed whether or not the non-treponemal test is reactive. ${ }^{22}$

This study formed part of a larger sero-prevalence study of HIV prevalence at Weskoppies Hospital ${ }^{29}$ - a specialist psychiatric hospital in Pretoria, which serves as a referral hospital for outlying clinics and secondary hospitals. The majority of inpatients at the hospital are involuntary mental healthcare users who suffer from serious mental illness such as schizophrenia, other psychotic disorders and, less frequently, mood disorders. In addition to the acute inpatient services, long-term inpatients undergo extended psychiatric rehabilitation, in some instances where their problems are of such a nature that previous attempts at community placement have failed. 
To contribute to the scant data on syphilis screening and prevalence in psychiatric patients, we aimed: (i) to determine and compare the prevalence of syphilis among different psychiatric inpatient groups; (ii) to determine and compare the psychiatric diagnoses of syphilisinfected and -uninfected patients; (iii) to assess self-reported high-risk sexual behaviour among patients; (iv) to establish the syphilis/HIV co-morbidity among patients; $(v)$ and to investigate the performance of the non-treponemal RPR test as a screening method compared with the confirmatory treponemal-specific TPHA test.

\section{Methods}

Consenting adult psychiatric inpatients $(N=195)$ were stratified into groups according to gender and length of admission ( $\geq 6$ months v. $\leq 3$ months), resulting in four categories: recently admitted male $(n=50)$; long-term male $(n=50)$, recently admitted female $(n=50)$; and longterm female $(n=45)$ patients. Stratified cluster sampling was used, with deliberately equal male:female and long-term:recent admission ratios. For a clear distinction between long-term and recent admissions, 3 - 6-month admissions were excluded. Cluster sampling was performed on randomly selected wards, on the premise that each ward was representative of the stratum to which it belonged. The smaller number of women tested was the result of a lack of long-term female patients who could give informed consent. Where necessary, weighted estimates were used to compensate for possible design bias in calculating syphilis prevalence.

\section{Statistical analysis}

A data capture sheet was used to record patients' demographic details, age, psychiatric diagnoses, self-reported high-risk sexual behaviour (e.g. multiple sexual partners, unprotected sexual intercourse with unknown partners, and prostitution) and intravenous drug use. The participants were screened for syphilis with the RPR and TPHA tests. A reactive TPHA test - indicating exposure to syphilis, although not necessarily active disease - was used to diagnose syphilis. HIV rapid (ACON HIV 1/2/0 Triline HIV Rapid Test Device) and fourth-generation HIV enzyme-linked immunosorbent assay (ELISA) tests were performed. ${ }^{29}$ The sensitivity and specificity, positive and negative predictive values, and likelihood ratios (LRs) for a positive (LR+) and negative (LR-) test were calculated to determine the screening performance of the RPR test. A cross-tabulation was constructed along with the odds ratio (OR) for syphilis prevalence. The sample was spliced post hoc according to psychiatric diagnosis, and analysed to determine differences with respect to syphilis prevalence. Data were analysed with SPSS software (version 17.0).

\section{Ethical considerations}

The ethical concerns regarding syphilis testing are not as stringent as those of HIV testing. Syphilis is less stigmatised and informed consent for serological testing is not a requirement. However, mentally ill patients may not comprehend information about the disease or issues related to testing, treatment and protecting sexual partners from infection. Nevertheless, psychosis per se does not exclude the provision of informed consent to participate in a research study. ${ }^{30}$

Patients were assessed clinically for their capacity to give informed consent for participation; those unable to give informed consent were excluded. Written informed consent was obtained following complete description of the study to the subjects. Participants were informed of their syphilis status, and managed with antimicrobial treatment in the case of a reactive syphilis test in the absence of a past history of syphilis treatment. For HIV testing, pre- and post-test counselling was performed according to regulations. Patients with reactive HIV tests were referred to the immunology clinic for management.

The study was approved by the Research Ethics Committee of the Faculty of Health Sciences, University of Pretoria.

\section{Results}

Of 195 patients tested, 23 were TPHA sero-positive (considered to be indicative of syphilis infection), while only 2 (1\%) had reactive RPR tests (with titres of 1:8 and 1:16, respectively) (Table 1). A syphilis prevalence of $11.7 \%$ ( $95 \%$ confidence interval (CI) 0.07 - 0.16) was estimated by calculating a weighted proportion based on the distribution of the strata in the population. There were no false-positive RPR tests.

Participant demographics, general characteristics, psychiatric diagnoses and TPHA sero-positivity are summarised in Table 2. Sero-positive TPHA results were found in 13/95 (14\%) women and 10/100 (10\%) men, and in more patients admitted for $\leq 3$ months (14\%) than those admitted for $\geq 6$ months (9\%). TPHA sero-positivity was similarly distributed among the subgroups, with the exception of a lower prevalence in the male long-term admission group (not significant). Psychotic disorders were the most common primary psychiatric diagnoses $(121 / 195 ; 62.1 \%)$ (Table 2). Mood disorders were the primary psychiatric diagnoses in 38/195 patients (19.5\%). There was no statistically significant association between primary psychiatric diagnosis and TPHA sero-positivity. Mood disorders tended to be more prevalent in patients with sero-positive TPHA results (not statistically significant). Psychotic disorders due to general medical conditions included 7 due to epilepsy (none with sero-positive syphilis results), and 6 attributed to HIV (2 with sero-positive TPHA results).

Eighty-five patients (44\%) had a secondary psychiatric diagnosis, most commonly cannabis abuse and/or dependence (21\%), mental retardation (12\%) and personality disorders (11\%). No secondary diagnosis was statistically associated with TPHA sero-positivity.

A large proportion of patients $(76 / 195 ; 39 \%)$ reported high-risk sexual behaviour, such as multiple partners, unknown partners or

\section{Table 1. RPR and TPHA results}

\begin{tabular}{lllll}
\hline & $\begin{array}{l}\text { Reactive } \\
\text { RPR test } \\
\text { Strata }\end{array}$ & RPR titre & $\begin{array}{l}\text { Reactive } \\
\text { TPHA } \\
\boldsymbol{n}(\%)\end{array}$ & $\begin{array}{l}\text { Number of } \\
\text { patients }\end{array}$ \\
\hline $\begin{array}{l}\text { Long-term admission } \\
\quad \text { Male }\end{array}$ & $0(0)$ & 0 & $3(6)$ & 50 \\
$\quad$ Female & $0(0)$ & 0 & $6(13)$ & 45 \\
Recent admission & & & & \\
$\quad$ Male & $1(2)$ & $1: 8$ & $7(14)$ & 50 \\
$\quad$ Female & $1(2)$ & $1: 16$ & $7(14)$ & 50 \\
Total, $N$ & $2(1)$ & 2 & $23(11.8)$ & 195 \\
RPR = rapid plasma reagin; TPHA $=$ T. pallidum haemagglutination test. &
\end{tabular}




\begin{tabular}{|c|c|c|c|}
\hline & $\begin{array}{l}\text { Reactive } \\
\text { TPHA } \\
n(\%)\end{array}$ & $\begin{array}{l}\text { Non-reactive } \\
\text { TPHA } \\
n(\%)\end{array}$ & $\begin{array}{l}\text { Number of } \\
\text { patients } \\
N\end{array}$ \\
\hline Age (years), mean $( \pm S D)$ & $41.7(8.8)$ & $38.7(12.3)$ & 39.1 \\
\hline \multicolumn{4}{|l|}{ Gender } \\
\hline Male & $10(10)$ & $90(90)$ & 100 \\
\hline Female & $13(14)$ & $80(86)$ & 95 \\
\hline \multicolumn{4}{|l|}{ Duration of admission } \\
\hline Recent (<3 months) & $14(14)$ & $86(86)$ & 100 \\
\hline Long-term (>6 months) & $9(9)$ & $86(91)$ & 95 \\
\hline \multicolumn{4}{|l|}{ Gender and duration } \\
\hline Male, recent & $7(14)$ & $43(86)$ & 50 \\
\hline Male, long-term & $3(6)$ & $47(94)$ & 50 \\
\hline Female, recent & $7(14)$ & $43(86)$ & 50 \\
\hline Female, long-term & $6(13)$ & $39(87)$ & 45 \\
\hline \multicolumn{4}{|l|}{ Sexual orientation } \\
\hline Heterosexual & $20(11)$ & 157 (89) & 177 \\
\hline Homosexual & $1(20)$ & $4(80)$ & 5 \\
\hline Bisexual & $2(15)$ & $11(85)$ & 13 \\
\hline \multicolumn{4}{|l|}{ Marital status } \\
\hline Married & $4(22)$ & $14(78)$ & 18 \\
\hline Unmarried & $19(11)$ & $158(89)$ & 177 \\
\hline \multicolumn{4}{|c|}{ Self-reported high-risk sexual behaviour } \\
\hline Yes & $7(9)$ & $69(91)$ & 76 \\
\hline No & $16(13)$ & $103(87)$ & 119 \\
\hline \multicolumn{4}{|l|}{ Primary psychiatric diagnosis } \\
\hline Schizophrenia & $10(11)$ & $77(89)$ & 87 \\
\hline Schizoaffective disorder & $2(7)$ & $27(93)$ & 29 \\
\hline $\begin{array}{l}\text { Psychotic disorder not } \\
\text { otherwise specified }\end{array}$ & $0(0)$ & $5(100)$ & 5 \\
\hline Bipolar disorder & $5(19)$ & $21(81)$ & 26 \\
\hline Major depressive disorder & $2(17)$ & $10(83)$ & 12 \\
\hline $\begin{array}{l}\text { Psychotic disorder due } \\
\text { to a general medical } \\
\text { condition }\end{array}$ & $2(15)$ & $11(85)$ & 13 \\
\hline $\begin{array}{l}\text { Substance-induced } \\
\text { psychotic disorder }\end{array}$ & $1(14)$ & $6(86)$ & 7 \\
\hline Dementia & $0(0)$ & $2(100)$ & 2 \\
\hline Mental retardation & $1(8)$ & $12(92)$ & 13 \\
\hline Personality disorder & $0(0)$ & $1(100)$ & 1 \\
\hline
\end{tabular}

absence of barrier protection. However, no statistically significant association was found between sero-positive syphilis results and self-reported high-risk sexual behaviour. Such behaviour was the lowest in the female recently admitted patients (28\%), and the highest in the male recently admitted group (46\%), with reported rates of $40 \%$ and $42 \%$ in the male and female long-term groups, respectively. The differences in high-risk sexual behaviour between groups were not statistically significant.

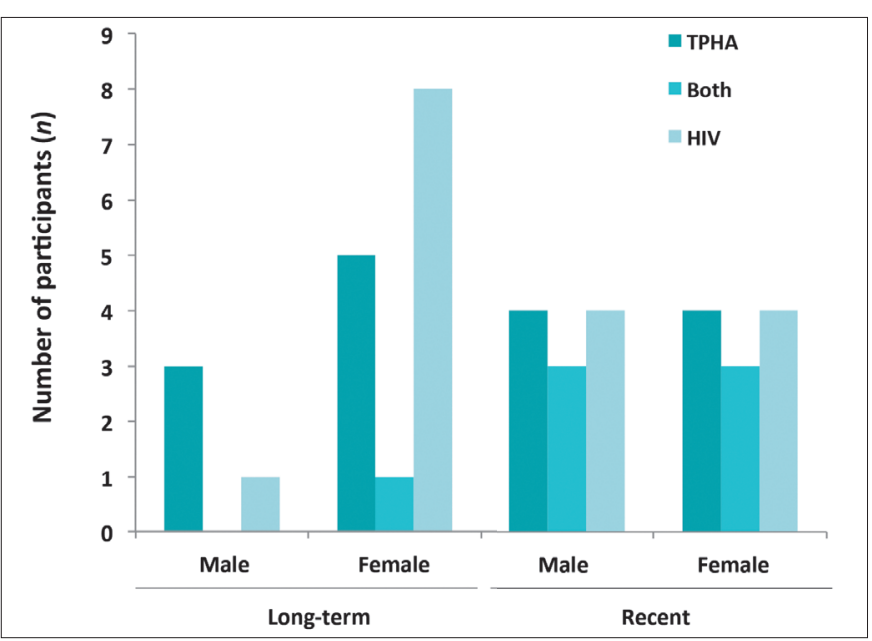

Fig. 1. Distribution of gender and length of admission (recent or long-term) in patients with syphilis (TPHA-positive) and/or HIV.

The co-morbidity of TPHA and HIV sero-positivity is illustrated in Fig. 1. Significantly, more patients who were diagnosed with syphilis also had reactive HIV results (Fisher's Exact test; $p=0.012$ ).

Concerning the performance of the RPR test as a diagnostic syphilis procedure, only $1 \%$ of patients had a reactive RPR test, whereas $11.8 \%$ had a sero-positive TPHA result (original figure, prior to estimation using weighted proportions). The RPR test had a sensitivity of only 0.087 (8.7\%) (95\% CI $0.05-0.13)$, a specificity of 1 (100\%), and no falsepositive results. Compared with the TPHA test, the RPR test performed poorly, identifying only $2 / 23$ patients who had syphilis. The positive predictive value of the RPR test was 1 (100\%), with a negative predictive value of 0.891 (89.1\%) and an LR- of 0.91 (91\%).

\section{Discussion}

To our knowledge, this is the first syphilis prevalence study in a state psychiatric hospital in SA. The $11.7 \%$ prevalence among psychiatric patients was lower than that found by other authors, but higher than anticipated. ${ }^{914}$ It is unknown whether the prevalence is comparable in local psychiatric hospitals. The statistical insignificance of the slightly lower syphilis prevalence in the male long-term group could reflect the high-risk sexual behaviour that was not statistically significantly different between the groups. The lack of statistical association between T. pallidum infection and psychiatric diagnostic profile is consistent with previous research, reaffirming syphilis as the great mimic of other psychiatric disorders. ${ }^{13}$ Concerning the secondary psychiatric diagnoses, the small number of patients with personality disorders and mental retardation may have prevented clear patterns from emerging.

Notwithstanding the substantial number of patients who reported high-risk sexual behaviour, no statistically significant association was found between such behaviour and sero-positive TPHA results, in contrast to previous research. ${ }^{13}$ This may be because high-risk sexual behaviour was self-reported.

We confirmed the well-established co-morbidity of HIV and syphilis infection. It is important to be aware of the HIV/syphilis co-morbidity and interaction: syphilis increases the risk of HIV infection and 
facilitates HIV transmission, whereas HIV alters the clinical picture and progression of the stages of syphilis.

The RPR test had an unacceptably low sensitivity as a syphilis screening procedure, indicating a low syphilis prevalence of only $1 \%$ (the TPHA test indicated a prevalence of $11.7 \%$ ). The use of nontreponemal tests only, as is current practice, would have resulted in missing positive TPHA results in $21 / 23$ patients in our cohort. The positive and negative predictive values and LR are difficult to interpret, given the very low syphilis prevalence associated with the RPR test. Nevertheless, it is clear that a positive RPR test on its own is a very poor syphilis screening tool.

This study provided much-needed insight into the sero-prevalence of syphilis in mentally ill, hospitalised patients in South Africa. Although research in this field is limited, our findings substantiated previous recommendations for the routine screening of psychiatric patients for syphilis. ${ }^{31}$ We demonstrated that the current practice of screening for syphilis is clearly inadequate in detecting syphilis exposure in all patients.

\section{Study limitations}

Statistical data analysis was limited by the small sample sizes of the four strata. It is also possible that some TPHA- and HIV-positive patients represented cases of biological false-positive TPHA, ${ }^{25}$ warranting further research on the incidence thereof. This should include a combination of molecular analysis, fluorescent treponemal antibody absorption (FTAabs), TPHA measurement at a higher dilution (to counter the effect of possible hypergammaglobulinaemia), total IgG, and screening for autoimmune diseases with anti-nuclear factor measurement.

\section{Conclusion}

The higher-than-anticipated $11.7 \%$ prevalence of syphilis in psychiatric inpatients at Weskoppies Hospital substantiates recommendations for routine testing. Both the RPR and TPHA tests should be performed in parallel, which increases the sensitivity dramatically. A substantial number of patients may not be diagnosed with syphilis with use of only the RPR test, as is current practice. Furthermore, taking into account the alarming prevalence and co-morbidity of HIV and syphilis, all psychiatric patients admitted should be tested for both diseases.

Acknowledgements. We are grateful to J Jordaan (Department of Statistics, University of Pretoria) for electronic data management, B English for language editing and M A Mabena (CEO, Weskoppies Hospital) for enabling research at the hospital. The study was financially supported by the Research Committee of the Faculty of Health Sciences, University of Pretoria. The authors have no conflicts of interest to declare.

\section{References}

1. Brandt AM. No magic bullet: A Social History of Venereal Diseases in the United States Since 1980. New York: Oxford University Press, 1987. [http://dx.doi org/10.1258/0956462021925081]

2. Yao Y, Huang E, Xie B, Cheng Y. Neurosyphilis presenting with psychotic symptoms and status epilepticus. Neurol Sci 2012;33(1):99-102. [http://dx.doi.org/10.1007/s10072-011-0563-y]
3. Zheng D, Zhou D, Zhao Z, et al. The clinical presentation and imaging manifestation of psychosis and dementia in general paresis: A retrospective study of 116 cases. J Neuropsychiatry Clinical Neurosci 2011;23(3):300-307. [http://dx.doi.org/10.1136/sextrans-2011-050247(2012)]

4. Roberts MC, Emsley RA. Psychiatric manifestations of neurosyphilis. S Afr Med J 1992;82(5):335337. [http://dx.doi.org/10.1016/j.psym.2012.07.002@2012]

5. French P. Syphilis - clinical review. BMJ 2007;334:143-137. [http://dx.doi.org/10.1136/ bmj.39085.518148.BE]

6. Kent E, Romanelli F. Re-examining syphilis: An update on epidemiology, clinical manifestations, and management. Ann Pharmacother 2008;42(2):226-236. [http://dx.doi.org/10.1345/aph.1 K086]

7. Tramont EC. Treponemapallidum. In: Mandell GL, ed. Mandell, Douglas and Bennett's Principles and Practice of Infectious Diseases. 4th ed. Edinburgh: Churchill Livingstone, 1995:2117-2131. [http://dx.doi.org/10:0443089353]

8. Timmermans M, Carr J. Neurosyphilis in the modern era. J Neurol Neurosurg Psychiatry 2004;75:1727-1730. [http://dx.doi.org/10.1136/jnnp.2004.031922]

9. Reeves RR, Pinkofsky HB, Kennedy KK. Unreliability of current screening tests for syphilis in chronic psychiatric patients. Am J Psychiatry 1996;153:1487-1488.

10. Zetola NM, Engelman J, Jensen TP, Klausner JD. Syphilis in the United States: An update for clinicians with an emphasis on HIV coinfection. Mayo Clin Proc 2007;82(9):1091-1102. [http:// dx.doi.org/10.4065/82.9.1091]

11. World Health Organization (WHO). Global Incidence and Prevalence of Selected Curable Sexually Transmitted Infections, 2008. Geneva: WHO, 2008. http://apps.who.int/iris/ handle/10665/75181:9789241503839_eng.pdf (accessed 4 August 2012)

12. Campos LN, Guimarães MD, Carmo RA, et al. HIV, syphilis, and hepatitis B and C prevalence among patients with mental illness: A review of the literature. Cad Saude Publica 2008;24(4):S607-S620. [http://dx.doi.org/10.1590/S0102-311X2008001600012]

13. Carey MP, Ravi V, Chandra PS, Desai A, Neal DJ. Prevalence of HIV, hepatitis B, syphilis, and chlamydia among adults seeking treatment for a mental disorder in southern India. AIDS Behav 2007;11(2):289-297. [http://dx.doi.org/10.1007/s10461-006-9134-2]

14. Hutto B, Adimora A. Syphilis in psychiatric inpatients: Prevalence, treatment, and implications. General Hospital Psychiatry 2000; 22(4):291-293. [http://dx.doi.org/10.1016/S0163-8343(00)000888]

15. Wilkinson D, Sach M, Connolly C. Epidemiology of syphilis in pregnancy in rural South Africa Opportunities for control. Trop Med Int Health 1997;2(1):57-62. [http://dx.doi.org/10.1136/ sti.2004.014068]

16. Ramjee G, Karim S, Sturm A. Sexually transmitted infections among sex workers in KwaZuluNatal, South Africa. Sex Transm Dis 1998;25(7):346-349. [http://dx.doi.org/10.1097/00007435199808000-00004]

17. Rottingen JA, Cameron DW, Garnett GP. A systematic review of the epidemiologic interaction between classic sexually transmitted diseases and HIV: How much is really known? Sex Transm Dis 2001;28:579-597.

18. Lynn WA, Lightman S. Syphilis and HIV: A dangerous combination. Lancet Infect Dis 2004;4:456466. [http://dx.doi.org/10.1016/S1473-3099(04)01061-8]

19. Buchacz K, Greenberg A, Onorato I, Janssen R. Syphilis epidemics and HIV incidence among men who have sex with men in the United States: Implications for HIV prevention. Sex Transm Dis 2005;32:S73-S79. [http://dx.doi.org/10.1097/01.olq0000180466.62579.4b]

20. Marra CM, Maxwell CL, Smith SL, et al. Cerebrospinal fluid abnormalities in patients with syphilis: Association with clinical and laboratory features. J Infect Dis 2004;189:369-376. [http://dx.doi. org/10.1086/381227]

21. Centers for Disease Control and Prevention. Sexually Transmitted Diseases Treatment Guidelines: Recommendations and Reports; no. RR-11. Morb Mortal Wkly Rep 2006;55:1-100. http://www.cdc. gov/std/treatment/2006/rr5511.pdf (accessed 23 November 2012).

22. Hutto B. Syphilis in clinical psychiatry: A review. Psychosomatics 2001;42:453-460. [http://dx.doi org/10.1176/appi.psy.42.6.453]

23. Workowski KA, Berman SM. Sexually Transmitted Diseases Treatment Guidelines, 2006. Recomm Rep 2006;55:1-94. http://www.cdc.gov/mmwr/preview/mmwrhtml/rr5511a1.htm (accessed 23 November 2012).

24. Larsen SA, Norris SJ, Pope V. Treponema and other host-associated treponemes. In: Murray PR, Baron EJ, eds. Manual of Clinical Microbiology. 7th ed. Washington, DC: American Society for Microbiology, 1999:759-768.

25. Ballard RC, Koornhof HJ, Chen C-Y, Radebe F, Fehler HG, Htun Y. The influence of concomitan HIV infection on the serological diagnosis of primary syphilis in southern Africa. S Afr Med I 2007;97(11):1151-1154.

26. Hooshmand H, Escabar MR, Kopf SW. Neurosyphilis, a study of 241 patients. JAMA 1972;219:726729. [http://dx.doi.org/10.1001/jama.1972.03190320032011]

27. Joyce CN, Molteno AC. Modified neurosyphilis in the Cape Peninsula. S Afr Med J 1978;53:10-14 [http://dx.doi.org/10.4103/0028-3886.53259]

28. Wöhrl S, Geusau A. Neurosyphilis is unlikely in patients with late latent syphilis and a negative blood VDRL-test. Acta Derm Venereol 2006;86:335-339. [http://dx.doi.org/10.2340/00015555-0092]

29. Henning MP, Krüger C, Fletcher L. HIV seropositivity in recently admitted and long-term psychiatric in-patients: Prevalence and diagnostic profile. Afr J Psychiatry 2012;15(1):47-53. [http:// dx.doi.org/10.4314/ajpsy.v15i1.7]

30. Van Staden CW, Krüger C. Incapacity to give informed consent owing to mental disorder. J Med Ethics 2003;29(1):41-43. [http://dx.doi.org/10.1136/jme.29.1.41]

31. Saik S, Kraus J, McDonald A, Mann S, Sheitman B. Neurosyphilis in newly admitted psychiatric patients: Three case reports. J Clin Psychiatry 2004;65(7):919-921. [http://dx.doi.org/10.1176/appi. psy.48.5.451] 